\title{
Supporting Information - Spin angular momentum of guided light induced by transverse confinement and intrinsic helicity
}

\author{
Diego R. Abujetas José A. Sánchez-Gil
}

Instituto de Estructura de la Materia (IEM-CSIC), Consejo Superior de Investigaciones

Científicas, Serrano 121, 28006 Madrid, Spain

\section{Electromagnetic fields for planar waveguides}

Let us consider a planar waveguide (see inset in Fig. 1a) consisting of a dielectric slab (core, medium 1) of thickness $2 d$ surrounded by another dielectric material (cladding, medium 2) that has a lower refractive index, $n_{1}=c \sqrt{\epsilon_{1} \mu_{1}}=\sqrt{\epsilon_{r 1} \mu_{r 1}}>n_{2}$, where $c=1 / \sqrt{\epsilon_{0} \mu_{0}}$ is the speed of light in vacuum. We define both relative and absolute dielectric permittivities and magnetic permeabilities $\left(\epsilon_{r}, \mu_{r}\right.$ and $\epsilon, \mu$, respectively). We assume propagation along the $z$ direction and translational invariance along the $y$ direction. Transverse electric (TE) and magnetic (TM) modes stand for modes with only electric/magnetic field component along the $y$ axis, propagating along the $z$ axis with propagation constant $k_{z}$ and transversal wavevector $k_{t}$. For TE modes, the corresponding fields (omitting the time harmonic factor $e^{-\imath \omega t}$ ) are [44]:

$$
\begin{aligned}
\mathbf{E} & =\hat{y} \sqrt{\mu_{i}} A_{i} f\left(k_{t} x\right) e^{\imath k_{z} z}, \\
\mathbf{H} & =A_{i} \frac{1}{\omega \sqrt{\mu_{i}}}\left[k_{z} f\left(k_{t} x\right) \hat{x}+\imath k_{t} f^{\prime}\left(k_{t} x\right) \hat{z}\right] e^{\imath k_{z} z},
\end{aligned}
$$

with $i=1,2$ and

$$
\begin{aligned}
f(x) & =e^{-\alpha x}, x>d, \\
& =\left\{\begin{array}{c}
\sin \left(k_{x} x\right) \\
\cos \left(k_{x} x\right)
\end{array}\right\},|x|<d, \\
& =\mp e^{\alpha x}, x<-d .
\end{aligned}
$$

The terms in braces denoting antisymmetric (top) and symmetric (bottom) modes, respectively; $f^{\prime}\left(k_{t} x\right)$ denotes derivative with respect to its argument. The electromagnetic fields for TM modes can be straightforwardly obtained from the above Eqs. (S1) by replacing $\mathbf{E}, \mathbf{H} \Rightarrow \mathbf{H},-\mathbf{E}$ and $\sqrt{\mu} \Rightarrow \sqrt{\epsilon}$. Field amplitudes $A_{i}$ outside/inside the waveguide are connected through corresponding boundary conditions. The wavevector $\mathbf{k}$ components in Cartesian coordinates of such fields depend also on the medium, and are given by:

$$
\mathbf{k}=\left(k_{t}, 0, k_{z}\right),
$$

with

$$
k_{t}= \pm \imath \alpha(|x|>d) \text { and } k_{t}=k_{x}(|x|<d),
$$

where we have used the same notation for the transversal component of the wavevector inside and outside the waveguide, bearing in mind that outside is complex, its sign $\imath \alpha$ depending on the considered semi-region. The wavevector components are related by:

$$
\begin{aligned}
& k_{z}^{2}+k_{t}^{2}=\epsilon_{r 1} \mu_{r 1}\left(\frac{\omega}{c}\right)^{2}=\left(n_{1} \frac{\omega}{c}\right)^{2},|x|>d, \\
& k_{z}^{2}-\alpha^{2}=\epsilon_{r 2} \mu_{r 2}\left(\frac{\omega}{c}\right)^{2}=\left(n_{2} \frac{\omega}{c}\right)^{2},|x|<d ;
\end{aligned}
$$

where $\omega$ is the angular frequency. Since the dielectric waveguide is intended to guide the light, the propagation constant $k_{z}$ has to be in the range $n_{2} / n_{1}<c k_{z} / \omega<n_{1}$ and will also depend on mode number and polarization.

\section{Electromagnetic fields for cylindrical waveguides}

Let us now study cylindrical waveguides, which support a wealth of guided modes exhibiting a rich spin phenomenology. The fields of the waveguide can be expressed in cylindrical coordinates [see inset in Fig. 1b] 
as follows:

$$
\begin{aligned}
& E_{r}=\sum\left[\frac{\imath k_{z}}{k_{t}} Z_{m}^{\prime}\left(k_{t} r\right) a_{m}-\frac{\mu \omega m}{k_{t}^{2} r} Z_{m}\left(k_{t} r\right) b_{m}\right] F_{m}, \\
& E_{\theta}=-\sum\left[\frac{m k_{z}}{k_{t}^{2} r} Z_{m}\left(k_{t} r\right) a_{m}+\frac{\imath \mu \omega}{k_{t}} Z_{m}^{\prime}\left(k_{t} r\right) b_{m}\right] F_{m}, \\
& E_{z}=\sum\left[Z_{m}\left(k_{t} r\right) a_{m}\right] F_{m}, \\
& H_{r}=\sum\left[\frac{m k^{2}}{\mu \omega k_{t}^{2} r} Z_{m}\left(k_{t} r\right) a_{m}+\frac{\imath k_{z}}{k_{t}} Z_{m}^{\prime}\left(k_{t} r\right) b_{m}\right] F_{m}, \\
& H_{\theta}=\sum\left[\frac{\imath k^{2}}{\mu \omega k_{t}} Z_{m}^{\prime}\left(k_{t} r\right) a_{m}-\frac{m k_{z}}{k_{t}^{2} r} Z_{m}\left(k_{t} r\right) b_{m}\right] F_{m}, \\
& H_{z}=\sum\left[Z_{m}\left(k_{t} r\right) b_{m}\right] F_{m},
\end{aligned}
$$

where $k_{z}$ and $k_{t}$ are again the longitudinal and transverse component component of the wavevector, respectively, and $k^{2}=(\mathbf{k} \cdot \mathbf{k})=\omega(\epsilon \mu)^{1 / 2}$ is the self dot product of the wavevector (analogous to Eqs. (S5)). Recall that $k_{t}$ is imaginary outside the waveguide. The function $Z_{m}\left(k_{t} r\right)$ is the appropriate Bessel function that matches the boundary conditions $[39,44]$ and $Z_{m}^{\prime}\left(k_{t} r\right)$ denotes the derivative with respect to the argument. Upon using functions $Z_{m}$, we collapse EM fields inside and outside the waveguide into formally identical equations. In this regard, recall that $a_{m}, b_{m}, \ldots$, obey different expressions inside/outside. The function $F_{m}=e^{\imath\left(m \theta+k_{z}-\omega t\right)}$ is the phase of the wave, the subscript $m$ being an integer related to its azimuthal order. Bear in mind that the longitudinal and transverse components of the wavevector depend on $m$, but we dropped the subscript for simplicity. For guided modes, the wavevector components are defined as in Eqs. (S5) (assuming vacuum outside $\epsilon_{r}=\mu_{r}=1$ ), replacing the transverse components $k_{x}$ and $\imath \alpha$ in Eq. (S3) by its transversal (radial) $k_{r}$ and $\imath \alpha$ counterparts associated with the cylindrical geometry.

The fields of the waveguide written in Eq (S6) are expressed as the sum over different guided modes, encoded in the subindex $m$. The guided modes satisfy the corresponding dispersion relation (not shown here, cf. Refs. $[38,39,42])$; solutions can be associated to each guided mode, labeled by a pair index $m l$, where $m=0, \pm 1, \pm 2, \ldots$ is the azimuthal index and $l=1,2,3, \ldots$ the radial index. For guided modes with $m=0$, the field is symmetric about the axis, exhibiting a pure transverse character, either electric $\left(\mathrm{TE}_{0 l}, a_{m}=0\right.$ so that $\left.E_{r}=E_{z}=H_{\theta}=0\right)$ or magnetic $\left(\mathrm{TM}_{0 l}, b_{m}=0\right.$ so that $\left.H_{r}=H_{z}=E_{\theta}=0\right)$. Hybrid modes arise for $m \neq 0\left(\mathrm{HE}_{m l}\right)$. which are the guided modes we will considered explicitly below.

\section{Energy, Poynting vector, and helicity densities in cylindrical waveguides}

The energy density is defined as:

$$
W=\frac{\epsilon}{4}|\mathbf{E}|^{2}+\frac{\mu}{4}|\mathbf{H}|^{2} .
$$

Upon introducing the electromagnetic fields of guided modes in a cylindrical lossless waveguide given by Eqs. (S6) into Eq. (S7), the resulting total energy density reads:

$$
\begin{aligned}
W= & \frac{1}{4}\left\{\left(\left|a_{m}\right|^{2} \epsilon+\left|b_{m}\right|^{2} \mu\right)\left[\left|Z_{m}\left(k_{t} r\right)\right|^{2}+\frac{k_{z}^{2}+k^{2}}{\left|k_{t}\right|^{2}}\left(\left|Z_{n}^{\prime}\left(k_{t} r\right)\right|^{2}+\frac{m^{2}}{\left|k_{t}\right|^{2} r^{2}}\left|Z_{m}\left(k_{t} r\right)\right|^{2}\right)\right]\right. \\
& \left.+\frac{8 m}{\left|k_{t}\right|^{2} r} \frac{k_{z} k}{k_{t}} Z_{m}\left(k_{t} r\right) Z_{m}^{\prime *}\left(k_{t} r\right) \Im\left[a_{m} b_{m}^{*}\right] \sqrt{\epsilon \mu}\right\} .
\end{aligned}
$$

The Poynting vector density,

$$
\mathbf{P}=\frac{1}{2 c^{2}} \Re\left[\mathbf{E}^{*} \times \mathbf{H}\right]=\frac{1}{2 c^{2}} \Re\left[\mathbf{E} \times \mathbf{H}^{*}\right]
$$

yields:

$$
\begin{aligned}
P_{r}= & 0 \\
P_{\theta}= & \frac{1}{2 \omega} \frac{1}{\epsilon_{r} \mu_{r}}\left\{\frac{m}{r} \frac{k^{2}}{k_{t}^{2}}\left|Z_{m}\left(k_{t} r\right)\right|^{2}\left(\left|a_{m}\right|^{2} \epsilon+\left|b_{m}\right|^{2} \mu\right)+2 \frac{k_{z} k}{k_{t}} Z_{m}^{\prime}\left(k_{t} r\right) Z_{m}^{*}\left(k_{t} r\right) \Im\left[a_{m} b_{m}^{*}\right] \sqrt{\epsilon \mu}\right\}, \\
P_{z}= & \frac{1}{2 \omega} \frac{1}{\epsilon_{r} \mu_{r}}\left\{\frac{k^{2}}{\left|k_{t}\right|^{2}} k_{z}\left(\left|a_{m}\right|^{2} \epsilon+\left|b_{m}\right|^{2} \mu\right)\left[\left|Z_{m}^{\prime}\left(k_{t} r\right)\right|^{2}+\frac{m^{2}}{\left|k_{t}^{2}\right| r^{2}}\left|Z_{m}\left(k_{t} r\right)\right|^{2}\right]\right. \\
& \left.+2 \frac{m}{r} \frac{k}{k_{t}} \frac{k_{z}^{2}+k^{2}}{\left|k_{t}\right|^{2}} Z_{m}\left(k_{t} r\right) Z_{m}^{*}\left(k_{t} r\right) \Im\left[a_{m} b_{m}^{*}\right] \sqrt{\epsilon \mu}\right\} .
\end{aligned}
$$

Finally, the helicity can be written as:

$$
h=-\frac{\sqrt{\epsilon_{0} \mu_{0}}}{2 \omega} \Im\left[\mathbf{E}^{*} \cdot \mathbf{H}\right] .
$$

For arbitrary guided mode in a cylindrical waveguide the helicity reads:

$$
\begin{aligned}
h= & \frac{1}{2 \omega n}\left\{\frac{2 m}{\left|k_{t}\right|^{2} r} \frac{k_{z} k}{k_{t}}\left[\left|a_{m}\right|^{2} \epsilon+\left|b_{m}\right|^{2} \mu\right] Z_{m}\left(k_{t} r\right) Z_{m}^{\prime *}\left(k_{t} r\right)\right. \\
& \left.+\left[\left|Z_{m}\left(k_{t} r\right)\right|^{2}+\frac{k_{z}^{2}+k^{2}}{\left|k_{t}\right|^{2}}\left(\left|Z_{n}^{\prime}\left(k_{t} r\right)\right|^{2}+\frac{m^{2}}{\left|k_{t}\right|^{2} r^{2}}\left|Z_{m}\left(k_{t} r\right)\right|^{2}\right)\right] \Im\left[a_{m} b_{m}^{*}\right] \sqrt{\epsilon \mu}\right\} .
\end{aligned}
$$


It can be shown that the energy and the helicity can be related as:

$$
h=\operatorname{sign}(m) \frac{W}{\omega n}-\frac{1}{4 \omega n} \tilde{F}_{a s y}(\mathbf{r})\left[\left|a_{m}\right| \sqrt{\epsilon}-\left|b_{m}\right| \sqrt{\mu}\right]^{2},
$$

where $\tilde{F}_{a s y}(\mathbf{r})$ is

$$
\tilde{F}_{a s y}(\mathbf{r})=\left|Z_{m}\left(k_{t} r\right)\right|^{2}+\frac{k_{z}^{2}+k^{2}}{\left|k_{t}\right|^{2}}\left[\left|Z_{n}^{\prime}\left(k_{t} r\right)\right|^{2}+\frac{m^{2}}{\left|k_{t}\right|^{2} r^{2}}\left|Z_{m}\left(k_{t} r\right)\right|^{2}\right]-4 m \frac{k_{z} k}{\left|k_{t}\right|^{2}} \frac{Z_{m}\left(k_{t} r\right) Z_{m}^{\prime}\left(k_{t} r\right)}{k_{t} r} .
$$

Using the next inequalities

$$
\begin{gathered}
\left|Z_{n}^{\prime}\left(k_{t} r\right)\right|^{2}+\frac{m^{2}}{\left|k_{t}\right|^{2} r^{2}}\left|Z_{m}\left(k_{t} r\right)\right|^{2} \geq 2 \frac{Z_{m}\left(k_{t} r\right) Z_{m}^{\prime}\left(k_{t} r\right)}{k_{t} r}, \\
k_{z}^{2}+k^{2} \geq 2 k_{z} k
\end{gathered}
$$

it can be seen that $\tilde{F}_{a s y}(\mathbf{r}) \geq 0$ and hence $|h| \leq W /(\omega n)$.

\section{Spin and momentum density in cylindrical waveguides}

The total spin angular momentum (SAM) density can be expressed as:

$$
\mathbf{S}=\frac{\epsilon_{0} \mu_{r}^{-1}}{4 \omega} \Im\left[\mathbf{E}^{*} \times \mathbf{E}\right]+\frac{\mu_{0} \epsilon_{r}^{-1}}{4 \omega} \Im\left[\mathbf{H}^{*} \times \mathbf{H}\right]
$$

For arbitrary guided modes in a cylindrical waveguide with electromagnetic field given by Eqs. (S6), the spin density obeys the following expressions:

$$
\begin{aligned}
S_{r}= & 0, \\
S_{\theta}= & \frac{1}{2 \omega} \frac{1}{\epsilon_{r} \mu_{r}}\left[\frac{k_{z}}{k_{t}} Z_{m}^{*}\left(k_{t} r\right) Z_{m}^{\prime}\left(k_{t} r\right)\left(\left|a_{m}\right|^{2} \epsilon+\left|b_{m}\right|^{2} \mu\right)\right. \\
& \left.+\frac{2 m}{k_{t}^{2} r} k\left|Z_{m}\left(k_{t} r\right)\right|^{2} \Im\left[a_{m} b_{m}^{*}\right] \sqrt{\epsilon \mu}\right], \\
S_{z}= & \frac{1}{2 \omega} \frac{1}{\epsilon_{r} \mu_{r}} \frac{1}{\left|k_{t}\right|^{2}}\left\{\frac{m}{k_{t} r} Z_{m}\left(k_{t} r\right) Z_{m}^{\prime *}\left(k_{t}\right)\left(k_{z}^{2}+k^{2}\right)\left(\left|a_{m}\right|^{2} \epsilon+\left|b_{m}\right|^{2} \mu\right)\right. \\
& \left.+2 k_{z} k\left[\left|Z_{m}^{\prime}\left(k_{t} r\right)\right|^{2}+\frac{m^{2}}{\left|k_{t}\right|^{2} r^{2}}\left|Z_{m}\left(k_{t} r\right)\right|^{2}\right] \Im\left[a_{m} b_{m}^{*}\right] \sqrt{\epsilon \mu}\right\},
\end{aligned}
$$

The spin momentum density,

$$
\mathbf{P}^{S}=\frac{1}{2} \nabla \times \mathbf{S}
$$

yields:

$$
\begin{aligned}
P_{r}^{S}= & 0, \\
P_{\theta}^{S}= & \frac{1}{4 \omega} \frac{1}{\epsilon_{r} \mu_{r}}\left\{\frac { m } { k _ { t } ^ { 2 } r } \left[k_{z}^{2}\left(\left|Z_{m}\left(k_{t} r\right)\right|^{2}-\frac{k_{t}^{2}}{\left|k_{t}\right|^{2}}\left(\left|Z_{m}^{\prime}\left(k_{t} r\right)\right|^{2}+\frac{m^{2}}{\left|k_{t}\right|^{2} r^{2}}\left|Z_{m}\left(k_{t} r\right)\right|^{2}-\frac{2}{k_{t} r} Z_{m}\left(k_{t} r\right) Z_{m}^{* *}\left(k_{t} r\right)\right)\right)\right.\right. \\
& \left.+k^{2}\left(\left(k_{t} r\right)^{-1} Z_{m}\left(k_{t} r\right) Z_{m}^{\prime *}\left(k_{t} r\right)-Z_{m}\left(k_{t} r\right) Z_{m}^{\prime \prime}\left(k_{t} r\right)-\frac{k_{t}^{2}}{\left|k_{t}\right|^{2}}\left|Z_{m}^{\prime}\left(k_{t} r\right)\right|^{2}\right)\right]\left[\left|a_{m}\right|^{2} \epsilon+\left|b_{m}\right|^{2} \mu\right] \\
& +2 k_{z} k\left[\frac{1}{\left|k_{t}\right|^{2} r}\left((m+1)\left|Z_{m}^{\prime}\left(k_{t} r\right)\right|^{2}+2 \frac{m^{2}}{\left|k_{t}\right|^{2} r^{2}}\left|Z_{m}\left(k_{t} r\right)\right|^{2}\right)\right. \\
& \left.\left.+k_{t}^{-1}\left(1-\frac{m(3 m+1)}{k_{t}^{2} r^{2}}\right) Z_{m}^{*}\left(k_{t} r\right) Z_{m}^{\prime}\left(k_{t} r\right)-k_{t}^{-1} Z_{m}^{\prime}\left(k_{t} r\right) Z_{m-1}^{\prime *}\left(k_{t} r\right)\right] \Im\left[a_{m} b_{m}^{*}\right] \sqrt{\epsilon \mu}\right\}, \\
P_{z}^{S}= & \frac{1}{4 \omega} \frac{1}{\epsilon_{r} \mu_{r}}\left\{k_{z}\left[\frac{k_{t}^{2}}{\left|k_{t}\right|^{2}}\left|Z_{m}^{\prime}\left(k_{t} r\right)\right|^{2}+\left(\frac{m^{2}}{k_{t}^{2} r^{2}}-1\right)\left|Z_{m}\left(k_{t} r\right)\right|^{2}\right]\left[\left|a_{m}\right|^{2} \epsilon+\left|b_{m}\right|^{2} \mu\right]\right. \\
& \left.+\frac{4 m k}{k_{t} r} Z_{m}^{*}\left(k_{t} r\right) Z_{m}^{\prime}\left(k_{t} r\right) \Im\left[a_{m} b_{m}^{*}\right] \sqrt{\epsilon \mu}\right\},
\end{aligned}
$$

Finally, the canonical (orbital) part $\mathbf{P}^{O}$ of the momentum density can be expressed (in cartesians coordinates) as:

$$
\mathbf{P}^{O}=\frac{\epsilon_{0} \mu_{r}^{-1}}{4 \omega} \Im\left[\mathbf{E}^{*} \cdot(\nabla) \mathbf{E}\right]+\frac{\mu_{0} \epsilon_{r}^{-1}}{4 \omega} \Im\left[\mathbf{H}^{*} \cdot(\nabla) \mathbf{H}\right],
$$

where we use the notation

$$
\left(\mathbf{X}^{*} \cdot(\nabla) \mathbf{Y}\right)_{i}=\sum_{i} X_{i}^{*} \frac{\partial}{\partial x_{i}} Y_{j}
$$


Bear in mind that Eq. (S22) is only valid in cartesians coordinates. The direct replacement of the $\nabla$ operator by its expression in other coordinates system is wrong. It is necessary to change the expression accordingly. After some algebraic manipulation we arrive to:

$$
\begin{aligned}
P_{r}^{O}= & 0, \\
P_{\theta}^{O}= & \frac{1}{4 \omega} \frac{1}{\epsilon_{r} \mu_{r}} \frac{1}{r}\left\{\frac{1}{\left|k_{t}\right|^{2}}\left|Z_{m}^{\prime}\left(k_{t} r\right)-\frac{m}{k_{t} r} Z_{m}\left(k_{t} r\right)\right|^{2}\left[m\left(k_{z}^{2}+k^{2}\right)\left[\left|a_{m}\right|^{2} \epsilon+\left|b_{m}\right|^{2} \mu\right]-4 k_{z} k \Im\left[a_{m} b_{m}^{*}\right] \sqrt{\epsilon \mu}\right]\right. \\
& +\frac{2 m(m-1)}{\left|k_{t}\right|^{2} k_{t} r} Z_{m}\left(k_{t} r\right) Z_{m}^{*}\left(k_{t} r\right)\left[\left(k_{z}^{2}+k^{2}\right)\left[\left|a_{m}\right|^{2} \epsilon+\left|b_{m}\right|^{2} \mu\right]+4 k_{z} k \Im\left[a_{m} b_{m}^{*}\right] \sqrt{\epsilon \mu}\right] \\
& \left.+m\left|Z_{m}\left(k_{t} r\right)\right|^{2}\left[\left|a_{m}\right|^{2} \epsilon+\left|b_{m}\right|^{2} \mu\right]\right\} \\
P_{z}^{O}= & \frac{1}{4 \omega} \frac{1}{\epsilon_{r} \mu_{r}} k_{z}\left\{\left[\left|Z_{m}\left(k_{t} r\right)\right|^{2}+\left[\left|Z_{m}^{\prime}\left(k_{t} r\right)\right|^{2}+\frac{m^{2}}{\left|k_{t}\right|^{2} r^{2}}\left|Z_{m}\left(k_{t} r\right)\right|^{2}\right] \frac{k_{z}^{2}+k^{2}}{\left|k_{t}\right|^{2}}\right]\left[\left|a_{m}\right|^{2} \epsilon+\left|b_{m}\right|^{2} \mu\right]\right. \\
& \left.+\frac{8 m}{\left|k_{t}\right|^{2} r} \frac{k_{z}}{k_{t}} Z_{m}\left(k_{t} r\right) Z_{m}^{*}\left(k_{t} r\right) \Im\left[a_{m} b_{m}^{*}\right] \sqrt{\epsilon \mu}\right\},
\end{aligned}
$$

which, upon comparing to the energy density (S8), it can be shown that the orbital momentum exhibits the expected dependence with the longitudinal wavevector $k_{z}$ :

$$
\frac{P^{O}}{W}=\frac{k_{z}}{\omega n^{2}} .
$$

\section{Orbital and Spin momenta in cylindrical waveguides for the hybrid $\mathrm{HE}_{11}$ mode}

We now turn to study the spin angular momentum of the $\mathrm{HE}_{11}$ hybrid guided mode, which in fact exhibit strong intrinsic helicity given by:

$$
\begin{aligned}
& h=\frac{1}{2 \omega n}\left\{\frac{2 k_{z} k}{\left|k_{t}\right|^{2} k_{t} r} Z_{1}\left(k_{t} r\right) Z_{1}^{\prime *}\left(k_{t} r\right)\left[\left|a_{1}\right|^{2} \epsilon+\left|b_{1}\right|^{2} \mu\right]\right. \\
& \left.+\left[\left|Z_{1}\left(k_{t} r\right)\right|^{2}+\left(\left|Z_{1}^{\prime}\left(k_{t} r\right)\right|^{2}+\frac{\left|Z_{1}\left(k_{t} r\right)\right|^{2}}{\left|k_{t}\right|^{2} r^{2}}\right) \frac{k_{z}^{2}+k^{2}}{\left|k_{t}\right|^{2}}\right] \Im\left[a_{1} b_{1}^{*}\right] \sqrt{\epsilon \mu}\right\}
\end{aligned}
$$

the corresponding energy density from Eq. (S8) is:

$$
\begin{aligned}
W=\frac{1}{4}\left\{\left[\left|Z_{1}\left(k_{t} r\right)\right|^{2}+\left(\left|Z_{1}^{\prime}\left(k_{t} r\right)\right|^{2}+\frac{\left|Z_{1}\left(k_{t} r\right)\right|^{2}}{\left|k_{t}\right|^{2} r^{2}}\right) \frac{k_{z}^{2}+k^{2}}{\left|k_{t}\right|^{2}}\right]\left[\left|a_{1}\right|^{2} \epsilon+\left|b_{1}\right|^{2} \mu\right]\right. \\
\left.+\frac{8 \sqrt{\epsilon \mu}}{\left|k_{t}\right|^{2}} \frac{k_{z} k}{k_{t} r} Z_{1}\left(k_{t} r\right) Z_{1}^{*}\left(k_{t} r\right) \Im\left[a_{1} b_{1}^{*}\right]\right\}
\end{aligned}
$$

and the spin density from Eq. (S18) is:

$$
\begin{aligned}
S_{r}= & 0, \\
S_{\theta}= & \frac{1}{2 \omega \epsilon_{r} \mu_{r}}\left[\frac{k_{z}}{k_{t}} Z_{1}^{*}\left(k_{t} r\right) Z_{1}^{\prime}\left(k_{t} r\right)\left[\left|a_{1}\right|^{2} \epsilon+\left|b_{1}\right|^{2} \mu\right]\right. \\
& \left.+\frac{2 k}{k_{t}^{2} r}\left|Z_{1}\left(k_{t} r\right)\right|^{2} \Im\left[a_{1} b_{1}^{*}\right] \sqrt{\epsilon \mu}\right], \\
S_{z}= & \frac{\left|k_{t}\right|^{-2}}{2 \omega \epsilon_{r} \mu_{r}}\left\{\frac{k_{z}^{2}+k^{2}}{k_{t} r} Z_{1}\left(k_{t} r\right) Z_{1}^{*}\left(k_{t} r\right)\left[\left|a_{1}\right|^{2} \epsilon+\left|b_{1}\right|^{2} \mu\right]\right. \\
& \left.+2 k_{z} k\left[\left|Z_{1}^{\prime}\left(k_{t} r\right)\right|^{2}+\frac{\left|Z_{1}\left(k_{t} r\right)\right|^{2}}{\left|k_{t}\right|^{2} r^{2}}\right] \Im\left[a_{1} b_{1}^{*}\right] \sqrt{\epsilon \mu}\right\},
\end{aligned}
$$

which becomes in this case very involved.

Finally, the orbital momentum from Eq. (S23) for the hybrid $\mathrm{HE}_{11}$ mode reads:

$$
\begin{aligned}
P_{r}^{O}= & 0 \\
P_{\theta}^{O}= & \frac{1}{4 \omega} \frac{1}{\epsilon_{r} \mu_{r}} \frac{1}{r}\left\{\frac{1}{\left|k_{t}\right|^{2}}\left|Z_{1}^{\prime}\left(k_{t} r\right)-\frac{Z_{1}\left(k_{t} r\right)}{k_{t} r}\right|^{2}\left[\left(k_{z}^{2}+k^{2}\right)\left[\left|a_{1}\right|^{2} \epsilon+\left|b_{1}\right|^{2} \mu\right]-4 k_{z} k \Im\left[a_{1} b_{1}^{*}\right] \sqrt{\epsilon \mu}\right]\right. \\
& \left.+\left|Z_{1}\left(k_{t} r\right)\right|^{2}\left[\left|a_{1}\right|^{2} \epsilon+\left|b_{1}\right|^{2} \mu\right]\right\}, \\
P_{z}^{O}= & \frac{1}{4 \omega} \frac{1}{\epsilon_{r} \mu_{r}} k_{z}\left\{\left[\left|Z_{1}\left(k_{t} r\right)\right|^{2}+\left[\left|Z_{1}^{\prime}\left(k_{t} r\right)\right|^{2}+\frac{\left|Z_{1}\left(k_{t} r\right)\right|^{2}}{\left|k_{t}\right|^{2} r^{2}}\right] \frac{k_{z}^{2}+k^{2}}{\left|k_{t}\right|^{2}}\right]\left[\left|a_{1}\right|^{2} \epsilon+\left|b_{1}\right|^{2} \mu\right]\right. \\
& \left.+\frac{8}{\left|k_{t}\right|^{2}} k_{z} k \frac{Z_{1}\left(k_{t} r\right) Z_{1}^{\prime}\left(k_{t} r\right)}{k_{t} r} \Im\left[a_{1} b_{1}^{*}\right] \sqrt{\epsilon \mu}\right\} .
\end{aligned}
$$


And the spin momentum, from Eq. (S20):

$$
\begin{aligned}
P_{r}^{S}= & 0, \\
P_{\theta}^{S}= & \frac{1}{4 \omega} \frac{1}{\epsilon_{r} \mu_{r}}\left\{\frac{1}{r}\left[\frac{\left|Z_{1}\left(k_{t} r\right)\right|^{2}}{k_{t}^{2}}-\frac{1}{\left|k_{t}\right|^{2}}\left|Z_{1}^{\prime}\left(k_{t} r\right)-\frac{Z_{1}\left(k_{t} r\right)}{k_{t} r}\right|^{2}\right]\left(k_{z}^{2}+k^{2}\right)\left[\left|a_{1}\right|^{2} \epsilon+\left|b_{1}\right|^{2} \mu\right]\right. \\
& \left.+4 k_{z} k\left[\frac{1}{\left|k_{t}\right|^{2} r}\left[\left|Z_{1}^{\prime}\left(k_{t} r\right)\right|^{2}+\frac{\left|Z_{1}\left(k_{t} r\right)\right|^{2}}{\left|k_{t}\right|^{2} r^{2}}\right]+\left(1-\frac{2}{k_{t}^{2} r^{2}}\right) \frac{Z_{1}^{*}\left(k_{t} r\right) Z_{1}^{\prime}\left(k_{t} r\right)}{k_{t}}\right] \Im\left[a_{1} b_{1}^{*}\right] \sqrt{\epsilon \mu}\right\}, \\
P_{z}^{S}= & \frac{1}{4 \omega} \frac{1}{\epsilon_{r} \mu_{r}}\left\{k_{z}\left[\frac{k_{t}^{2}}{\left|k_{t}\right|^{2}}\left|Z_{1}^{\prime}\left(k_{t} r\right)\right|^{2}+\left(\frac{1}{k_{t}^{2} r^{2}}-1\right)\left|Z_{1}\left(k_{t} r\right)\right|^{2}\right]\left[\left|a_{1}\right|^{2} \epsilon+\left|b_{1}\right|^{2} \mu\right]\right. \\
& \left.+\frac{4}{r} \frac{k}{k_{t}} Z_{1}^{*}\left(k_{t} r\right) Z_{1}^{\prime}\left(k_{t} r\right) \Im\left[a_{1} b_{1}^{*}\right] \sqrt{\epsilon \mu}\right\} .
\end{aligned}
$$



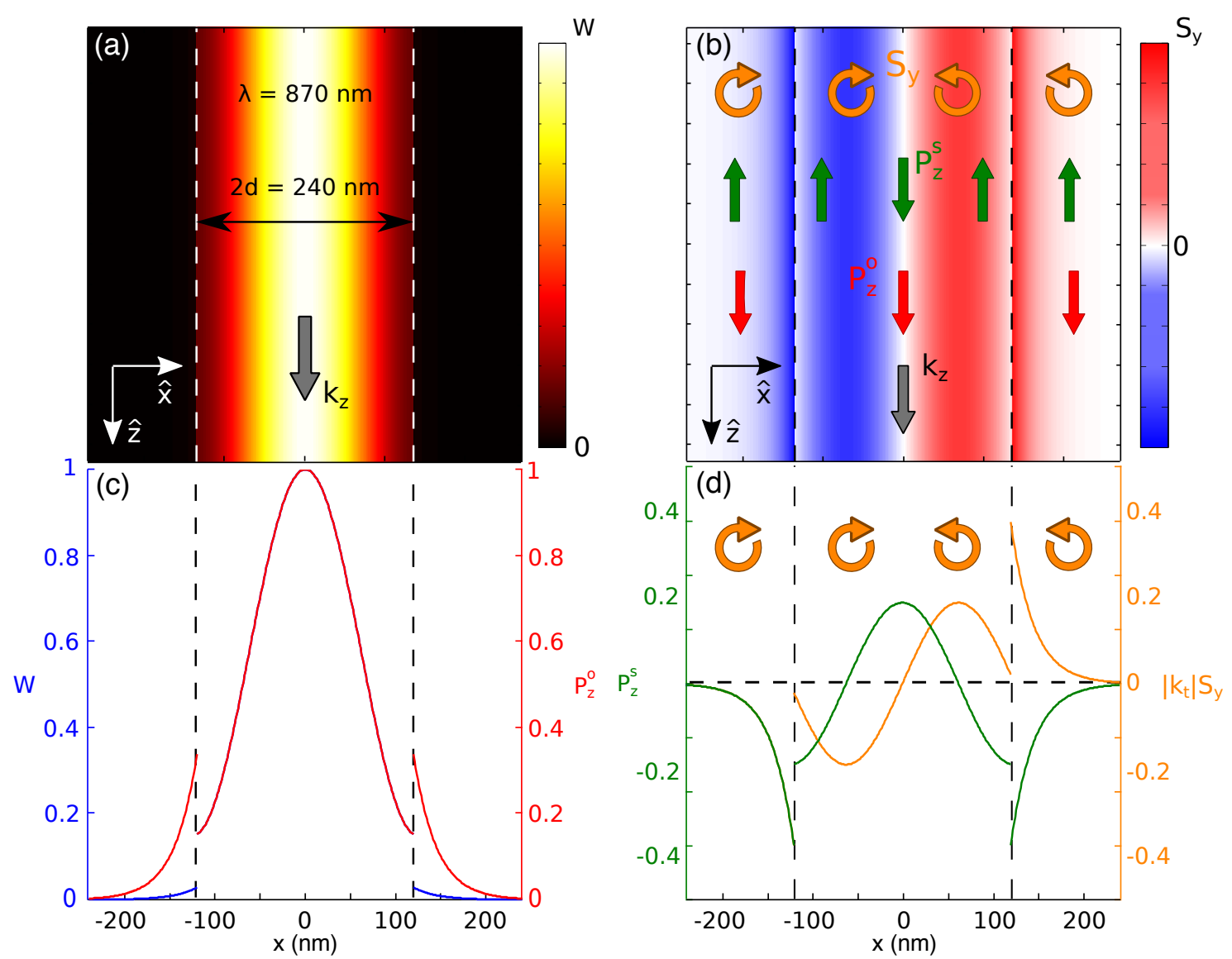

Figure S1. (a-d) The symmetric lowest-order TM mode is considered for a planar waveguide of width $2 d$ and $\epsilon_{r}=11.76$ for normalized half-width $\omega d / c=0.87$ (which corresponds to an InP nanoslab with thickness $2 d=240 \mathrm{~nm}$ at $\lambda=870 \mathrm{~nm}$ ). (a,c) Contour map and radial dependence of the energy density $W$, the latter including also the orbital $P_{z}^{O}$ momentum. (b,d) Contour map and radial dependence of the only non-zero component of the spin density $S_{y}$, the latter including also the only non-zero component of the spin momentum $P_{z}^{S}$. 

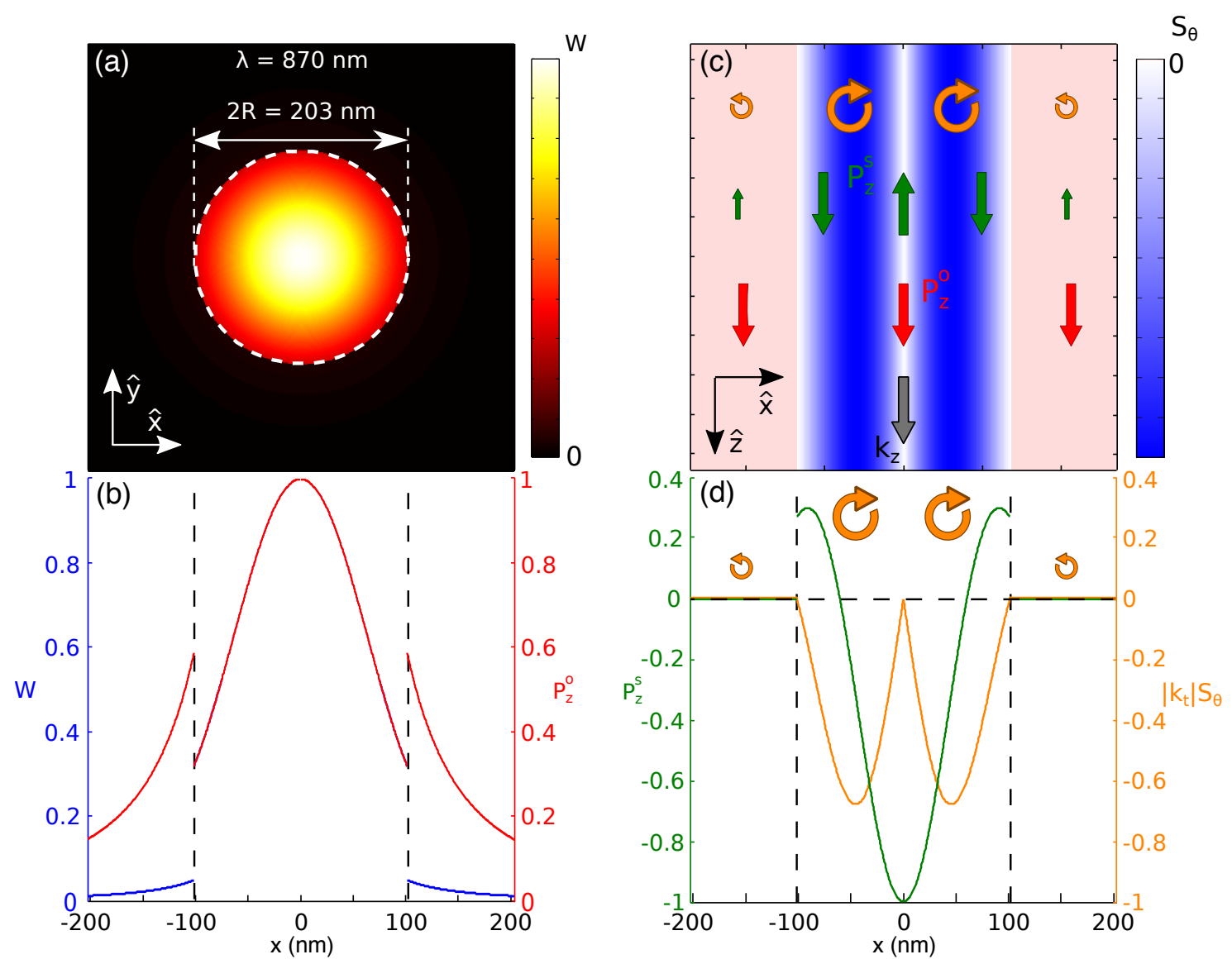

Figure S2. (a-d) The lowest-order $\mathrm{TE}_{01}$ guided mode for a cylindrical waveguide of radius $R$ and $\epsilon_{r}=11.76$ is considered for a normalized radius $\omega R / c=0.73$ (which corresponds to an InP nanowire with $2 R=203$ $\mathrm{nm}$ at $\lambda=870 \mathrm{~nm}$, near by the cutoff frequency): (a,c) Contour map and radial dependence of the energy density $W$, the latter including also the orbital $P_{z}^{O}$ momentum. (b,d) Contour map and radial dependence of the only non-zero component of the spin density $S_{\theta}$, the latter including also the only non-zero component of the spin momentum $P_{z}^{S}$. 

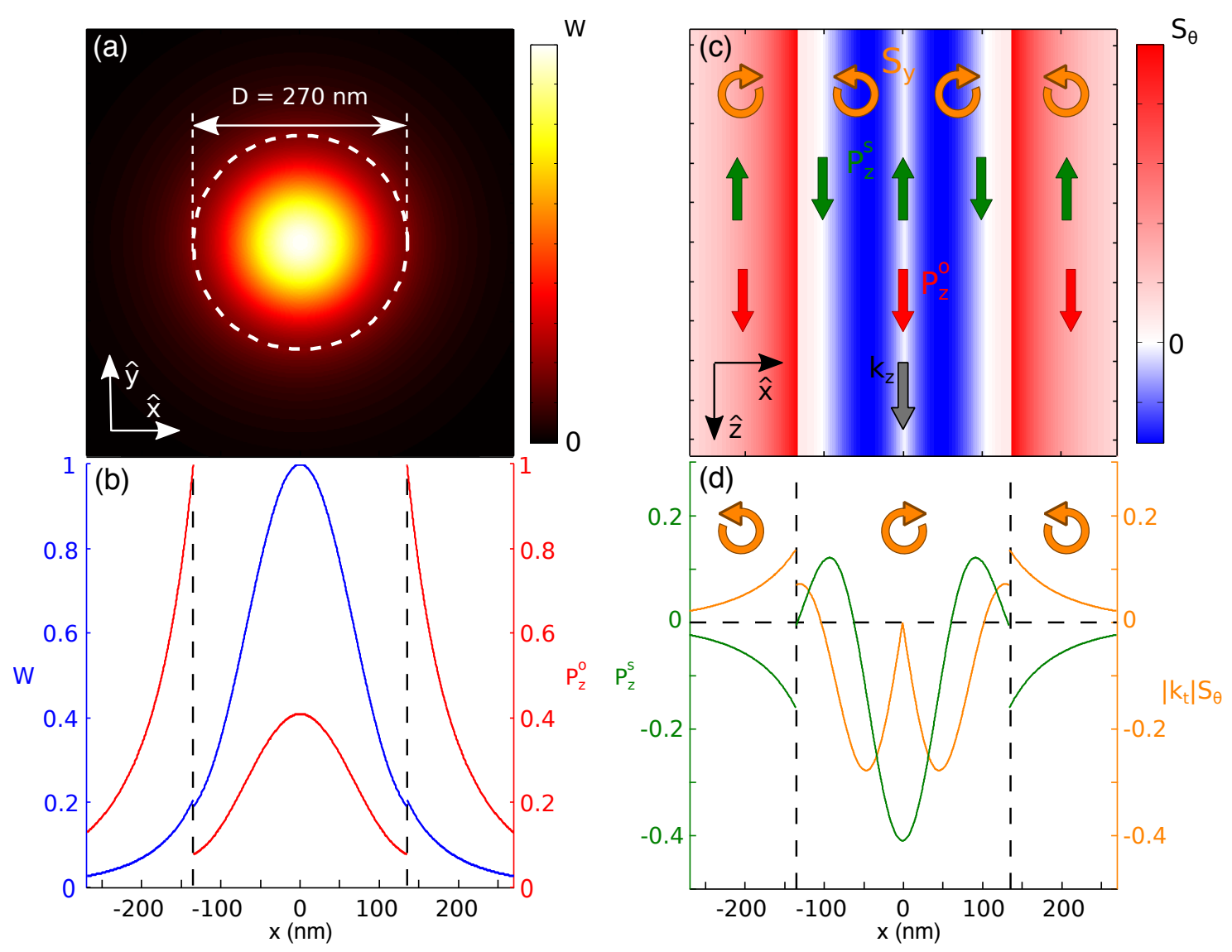

Figure S3. (a-d) The lowest-order $\mathrm{TM}_{01}$ guided mode for a cylindrical waveguide of radius $R$ and $\epsilon_{r}=11.76$ is considered for a normalized radius $\omega R / c=0.97$ (which corresponds to an InP nanowire with $2 R=270$ $\mathrm{nm}$ at $\lambda=870 \mathrm{~nm}$ ): (a,c) Contour map and radial dependence of the energy density $W$, the latter including also the orbital $P_{z}^{O}$ momentum. (b,d) Contour map and radial dependence of the only non-zero component of the spin density $S_{\theta}$, the latter including also the only non-zero component of the spin momentum $P_{z}^{S}$. 